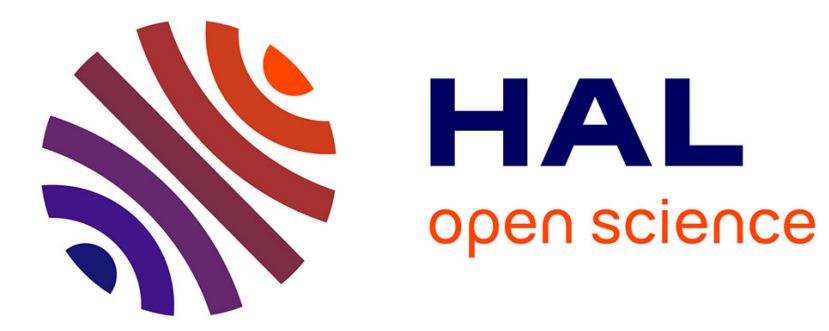

\title{
Electro-optic effect exaltation on lithium niobate photonic crystals due to slow photons
}

M. Roussey, M.-P. Bernal, N. Courjal, D. van Labeke, F.I. Baida, R. Salut

\section{To cite this version:}

M. Roussey, M.-P. Bernal, N. Courjal, D. van Labeke, F.I. Baida, et al.. Electro-optic effect exaltation on lithium niobate photonic crystals due to slow photons. Applied Physics Letters, 2006, 89, pp.241110. 10.1063/1.2402946 . hal-00175598

\section{HAL Id: hal-00175598 \\ https://hal.science/hal-00175598}

Submitted on 12 Apr 2021

HAL is a multi-disciplinary open access archive for the deposit and dissemination of scientific research documents, whether they are published or not. The documents may come from teaching and research institutions in France or abroad, or from public or private research centers.
L'archive ouverte pluridisciplinaire HAL, est destinée au dépôt et à la diffusion de documents scientifiques de niveau recherche, publiés ou non, émanant des établissements d'enseignement et de recherche français ou étrangers, des laboratoires publics ou privés. 


\title{
Electro-optic effect exaltation on lithium niobate photonic crystals due to slow photons
}

\author{
M. Roussey, M.-P. Bernal, ${ }^{\text {a) }}$ N. Courjal, D. Van Labeke, and F. I. Baida \\ Département LOPMD, Institut FEMTO-ST (UMR 6174), 16 Route de Gray, 25030-Besançon Cedex, France \\ R. Salut \\ Centrale de Technologie MIMENTO, Institut FEMTO-ST (UMR 6174), 32 Avenue de l'Observatoire, 25030- \\ Besançon Cedex, France
}

(Received 18 August 2006; accepted 20 October 2006; published online 12 December 2006)

\begin{abstract}
The authors demonstrate how slow group velocities that are easily attainable at the band edge of photonic crystals can drastically enhance the electro-optical effect on tunable photonic crystal components. This property opens up the possibility of microsized nonlinear devices with low power requirement. In this letter we show how these possibilities for enhancement of nonlinear effects have been used to fabricate a $13 \times 13 \mu \mathrm{m}^{2}$ sized lithium niobate photonic crystal intensity modulator that shows an enhanced electro-optic effect 312 times bigger than the one predicted by the classical Pockels effect for an equivalent device in bulk material. (C) 2006 American Institute of Physics.
\end{abstract} [DOI: $10.1063 / 1.2402946$ ]

During the last years, there has been a great interest in photonic crystals (PCs) due to their ability to manipulate photons and to their potential applications in photonic information technology. ${ }^{1}$ By replacing standard optical devices by the photonic crystal equivalent optical circuit, a considerable size and optical loss reduction can be achieved, leading to micrometric switches, modulators, or reconfigurable networks. In the perspective of integrating dense optical circuits on small surfaces, tunable PCs present special interest. They typically consist of a periodic array of air holes on a dielectric substrate whose optical properties are modified by an external physical signal (electric or magnetic field, temperature, strain, etc.). ${ }^{2-15}$ One of the most suitable tuning schemes may be based on the application of an electric field, due to the technical compatibility of the PC components with current microelectronic technology. Indeed, one of the most promising tunable $\mathrm{PC}$ configurations is a polymer-based photonic device tuned by the Pockels effect showing sub-1-V sensitivity. ${ }^{9}$

However, up to now, electro-optically tunable PCs have limited tunability because of the small attainable changes in the refractive index although these small changes are suitable to realize electro-optic photonic crystal superprism devices. ${ }^{16}$ In this letter we demonstrate that the electro-optic tunability of a PC can be drastically enhanced if the device operates at low group velocities.

It is well known that nonlinear effects can be enhanced in systems with slow group velocity as a result of the compression of the local density of states. ${ }^{1}$ For this purpose, some theoretical and experimental studies have already been published. ${ }^{17-20}$ An interesting application has appeared recently, exploiting the slow light phenomena in PC devices to enhance Kerr and four-wave mixing in a one-dimensional structure. ${ }^{21}$ However, no experimental application has appeared so far putting in evidence that slow light can also be used to enhance the second order nonlinear susceptibility in a two-dimensional (2D) photonic crystal. In this letter, slow light phenomenon is directly exploited to enhance the electro-optic tunability of a lithium niobate photonic crystal.

\footnotetext{
${ }^{a)}$ Electronic mail: maria-pilar.bernal@univ-fcomte.fr
}

The choice of lithium niobate as substrate material is motivated because of its large electro-optic, acousto-optic, and nonlinear optical coefficients. In addition, it has extensive applications in piezoelectric, acousto-optic, nonlinear, and photorefractive devices.

We have fabricated lithium niobate (LN) photonic crystals and shown an experimental realization of a LN photonic band gap structure. ${ }^{22,23}$ One additional step is attained in this work towards the fabrication of multitunable, ultracompact photonic crystals with the experimental demonstration of a low-voltage, micrometric-sized electro-optic LN photonic crystal intensity modulator. The modulator, exhibits wavelength shift sensitivity 312 times larger than what is predicted by the classical Pockels effect applied to a nonstructured LN substrate. The measured shift corresponds to a refractive index variation of 0.3 for an applied external voltage of $80 \mathrm{~V}$. With an active length of only $11 \mu \mathrm{m}$, our device exhibits sensitivity 1000 times higher than state of the art electro-optical tunable photonic crystals. ${ }^{9}$

The device consists of a $15 \times 15$ squared array of air holes etched by focused ion beam (FIB) on a gradient index $\mathrm{LN}$ waveguide. The fabrication details of the structure are described in detail in a previous work. ${ }^{23}$ An alternative fabrication technique that consists of electric poling and subsequent etching has also been reported elsewhere. ${ }^{24}$ The geometrical parameters of the PC are fixed by theoretical calculations so that an edge of the gap corresponds to the operating wavelength of $1550 \mathrm{~nm}$. The dispersion relationship for the first Brillouin zone is shown in Fig. 1(a). As we can see, the $\Gamma X$ direction presents a flat band edge that gives rise to low group velocity propagating conditions. In what follows we shall see that if we design our device in this band edge operating zone, slow photons are going to enhance lithium niobate nonlinear effects, in particular, the Pockels effect.

By using two-dimensional finite difference time domain (2D-FDTD) homemade calculations, we have determined that a squared arrangement of holes with a period $a$ $=766 \mathrm{~nm}$ and $r / a=0.27$ ratio ( $r$ being the radius of the hole) induces a gap with an edge at $1550 \mathrm{~nm}$ in the $\Gamma X$ direction of propagation and TE polarization [see Figs. 1(b) and 1(c)]. An 


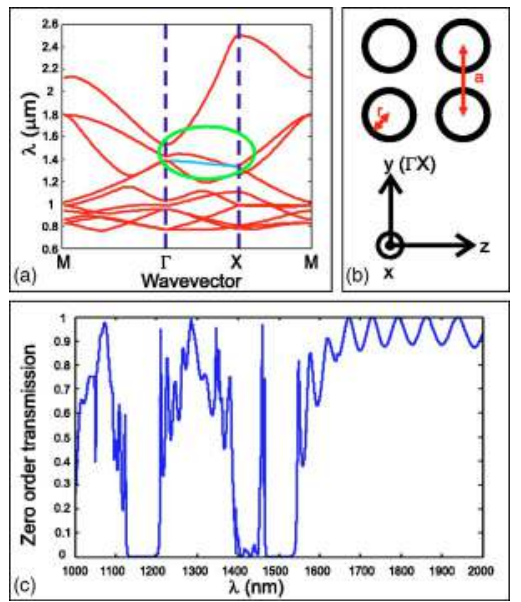

FIG. 1. (Color online) (a) Band diagram for the first Brillouin zone in a squared lattice lithium niobate photonic crystal. (b) Schematic of the simulated structure that consists of $15 \times 15$ holes arranged in a squared lattice. (c) Numerical simulation of the transmitted intensity through the photonic crystal structure performed by 2D-FDTD method.

interesting feature in the transmission spectrum [Fig. 1(c)] is the presence of a peak that could also be modulated at $1460 \mathrm{~nm}$. An index variation is induced by the Pockels effect using coplanar electrodes deposited on the structure. In order to benefit from the largest electro-optical coefficient of the LN substrate, which is $r_{33}=30.8 \mathrm{pm} / \mathrm{V}$ at $1550 \mathrm{~nm}$, the electrodes are disposed at both sides and parallel to the optical waveguide. The wafer is cut normally to the $x$ axis, and the propagation of the light follows the $y$ axis [see Fig. 1(b)]. By injecting a TE-polarized light into the photonic structure, a maximum index variation can be obtained. By applying the classical Pockels formula to lithium niobate, a 0.01 index variation should typically correspond to a $67.10 \mathrm{~V} / \mu \mathrm{m}$ electric field and an $863 \mathrm{~V}$ applied voltage. In what follows, we show that the photonic structure at the band edge of the dispersion curve lowers considerably the expected driving voltage.

Figures 2(a) and 2(b) show the scanning electron microscope (SEM) image of the photonic crystal and a photograph of the fabricated tunable device, respectively. The electrodes have been fabricated by depositing $150 \mathrm{~nm}$ of Ti by sputtering (Alcatel SCM 450). The distance between both electrodes is $13 \mu \mathrm{m}$ and their length is $8 \mathrm{~mm}$. The LN sample containing the electrodes and the photonic crystal is placed in a butterfly shaped electronic circuit to facilitate the electrical connection. On the circuit, two copper lines have been traced and the Ti electrodes are connected to them through wire bonding.

In order to characterize the transmission spectrum, a white light source was employed. The white light is generated by a subnanosecond microchip laser emitting light at
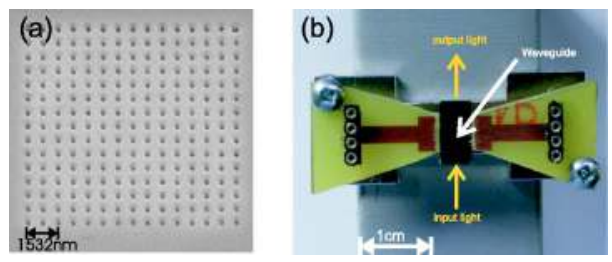

FIG. 2. (Color online) (a) Scanning electron microscope (SEM) image of the fabricated squared lattice lithium niobate photonic crystal. (b) Picture of the electro-optic tunable photonic crystal device.

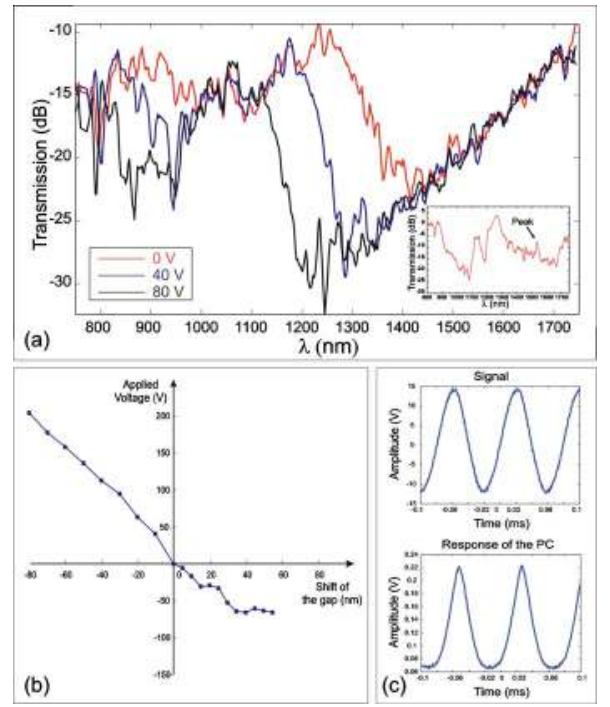

FIG. 3. (Color online) (a) Measured transmission of the photonic crystal for different applied external voltages $(0,40$, and $80 \mathrm{~V})$. Inset: Measured transmission of a different LN substrate with the same geometry, where the central peak has been observed. (b) Wavelength shift measured for a $\lambda$ $=1300 \mathrm{~nm}$ at $0 \mathrm{~V}$ as a function of external positive and negative voltages. (c) Modulation performance of the photonic crystal. The input is a sinusoidal signal at $13.5 \mathrm{~V}$ and $10 \mathrm{kHz}$. The output transmitted signal presents an extinction ratio of $5.2 \mathrm{~dB}$. The measurement has been performed at a wavelength of $1550 \mathrm{~nm}$ for which the waveguide presents monomodal behavior.

$1064 \mathrm{~nm}$ with $8 \mu \mathrm{J} /$ pulse. The laser light is coupled into $20 \mathrm{~m}$ of photonic crystal fiber, which enhances the nonlinear effects required for the generation of a large band supercontinuum $[600,1800] \mathrm{nm}$. In the first experiment, the electrodes are connected to a continuum voltage generator. The schematic of the experiment is the same as in Ref. 23.

The transmission spectrum for different excitation voltages is shown in Fig. 3(a). At $0 \mathrm{~V}$ two consecutive stop bands are observed. Their location corresponds to the theoretical prediction (first band $[1125,1200] \mathrm{nm}$, second band $[1400,1550] \mathrm{nm})$ although the measured stop bands are wider due to fabrication imperfections. However, no peak has been measured in this particular sample although we have experimentally observed it in other samples presenting the same geometry [see inset on Fig. 3(a)]. The absence of a peak can be attributed to imperfections in the geometry that has been confirmed by numerical simulations. Indeed, we have calculated the transmission spectrum when the holes are slightly misaligned, obtaining a stop gap without any peak. Given the resolution of a FIB (around $50 \mathrm{~nm}$ ), it is possible that the absence of the measured peak in this particular sample is due to a slight misalignment of the FIB during the etching process.

When a continuous voltage is applied, the band gap shifts [Fig. 3(a)]. The measured wavelength shift is $2.5 \mathrm{~nm} / \mathrm{V}$. This value is 312 times bigger than the shift predicted by the Pockels equation in bulk $(0.008 \mathrm{~nm} / \mathrm{V})$. The band shift changes direction as we invert the applied voltage sign, confirming an electric effect [Fig. 3(b)]. To rule out photorefractive additional effects we have repeated the same experiment with the sample continuously illuminated by a blue laser beam $(50 \mathrm{~mW}, \lambda=473 \mathrm{~nm})$, where photorefractive effect is most significant for lithium niobate, ${ }^{25}$ over $3 \mathrm{~h}$. No additional effect was appreciated. It is necessary to point out that index variations due to photorefractive effect are gener- 
ally three orders of magnitude smaller than the index variation reported here $(\Delta n=0.3)$.

To quantify this extraordinary Pockels effect, we have calculated the local field inside the photonic crystal device following the same calculations as in Ref. 21 but for a 2D photonic crystal. The local field factor is directly calculated with the group velocity inside the bulk LN substrate, $\nu_{g}^{\text {bulk }}$, and the group velocity in the photonic crystal $\nu_{g}^{\mathrm{PC}}$,

$$
f=\sqrt{\frac{\nu_{g}^{\text {bulk }}}{\nu_{g}^{\mathrm{PC}}}} .
$$

The local field at the lower band edge of the dispersion relation attains a value of 7 for $\lambda=1400 \mathrm{~nm}$. For this particular wavelength the group velocity inside the photonic crystal is $2.86 \times 10^{6} \mathrm{~m} / \mathrm{s}$, that is, 49 times smaller than in bulk lithium niobate. Taking into account that the effective second order susceptibility (for the photonic crystal) is obtained:

$$
\chi_{\mathrm{PC}}^{(2)}=f_{1} f_{2} f_{3} \chi^{(2)},
$$

where $\chi^{(2)}$ is the intrinsic second order susceptibility of lithium niobate (the PC substrate) and $f_{i}(i=1,3)$ are the local field factors for the three waves involved in the second order process.

Given that the electro-optical coefficient $r_{33}$ is, in first approximation, proportional to $\chi^{(2)}$, we can define an effective electro-optical effect as

$$
r_{33}^{\mathrm{PC}}=f_{1} f_{2} f_{3} r_{33} .
$$

For $\lambda=1400 \mathrm{~nm}$, and taking into account that $r_{33}$ $=32 \mathrm{pm} / \mathrm{V}$, the electro-optic coefficient in the photonic crystal is then equal to $r_{33}^{\mathrm{PC}}=10564 \mathrm{pm} / \mathrm{V}$. The refractive index difference is then obtained with the Pockels formula as follows:

$$
\Delta n=-\frac{1}{2} r_{33}^{\mathrm{PC}} n_{e}^{3} E_{z},
$$

where $n_{e}$ denotes the extraordinary refractive index and $E_{z}$ is the mean $z$ component of the electric field applied on the photonic crystal structure.

If Eq. (4) is applied for the lower band edge of the band gap $(\lambda=1400 \mathrm{~nm})$ and an external voltage of $80 \mathrm{~V}$, we obtain a $\Delta n=0.32$, in excellent agreement with the experimental measurements.

Note that the right band edge does not shift as much as the lower band edge. As we see in the band diagram [Fig. 1(a)], the right band edge is not flat, giving rise to nonzero values for the group velocity.

An ultracompact LN electro-optic modulator has been experimentally exploited based on the LN photonic crystal structure previously described. The modulation performance of this fabricated modulator has been studied and the result of the modulated signal is displayed in Fig. 3(c). A sinusoidal input signal is applied to the electrodes with a driving voltage of $13.5 \mathrm{~V}$ at $10 \mathrm{kHz}$. A laser light at $1550 \mathrm{~nm}$ (for which the LN waveguide is monomode) has been injected to the structure. The transmitted output signal has a sinusoidal shape that follows the input signal. An extinction ratio of
$5.2 \mathrm{~dB}$ has been measured. The length of the modulator is only $13 \mu \mathrm{m}$ as opposed to the several millimeters exhibited by standard LN modulators (Ref. 26).

In conclusion, the stop band of a square lattice LN photonic crystal has been shifted with an external voltage. Unexpectedly, the experimentally measured shift is 312 times bigger than the one predicted by the Pockels effect, which is due to slow light effects that enhance the second order susceptibility of the lithium niobate photonic crystal. This photonic crystal has been used as an ultracompact, low-voltage LN intensity modulator.

This work has been supported by the Action Concertée Incitative "Nanosciences" COBIAN, No. NR137. The authors would like to acknowledge the technical assistance provided by MIMENTO, FEMTO-ST, Yannick Gruson for the wire bonding, and particularly Eloïse Devaux from the Laboratoire des Nanostructures ISIS, Université Louis Pasteur, Strasbourg for the FIB fabrication of the structures.

${ }^{1}$ Y. A. Vlasov, M. O'Boyle, H. F. Hamann, and S. J. McNab, Nature (London) 438, 65 (2005).

${ }^{2}$ B. Li, J. Zhou, L. Li, X. J. Wang, X. H. Liu, and J. Zi, Appl. Phys. Lett. 83, 4704 (2003).

${ }^{3}$ H. G. Park, S. H. Kim, S. H. Kwon, Y. G. Ju, J. K. Yang, J. H. Baek, S. B. Kim, and Y. H. Lee, Science 305, 1444 (2004).

${ }^{4}$ H. Takeda and K. Yoshino, Phys. Rev. E 69, 016605 (2004).

${ }^{5}$ S. P. Gorkhali, J. Qi, and G. P. Grawford, Appl. Phys. Lett. 86, 011110 (2005).

${ }^{6}$ Y. Jiang, W. Jiang, L. Gu, X. Chen, and R. T. Chen, Appl. Phys. Lett. 87, 221105 (2005).

${ }^{7}$ S. L. Kuai, G. Bader, and P. V. Ashrit, Appl. Phys. Lett. 86, 221105 (2005).

${ }^{8}$ D. McPhail, M. Straub, and M. Gu, Appl. Phys. Lett. 86, 051103 (2005).

${ }^{9}$ M. Schmidt, M. Eich, U. Huebner, and R. Boucher, Appl. Phys. Lett. 87, 121110 (2005).

${ }^{10}$ H. M. H. Chong and R. M. De La Rue, IEEE Photonics Technol. Lett. 16, 1528 (2004).

${ }^{11}$ B. Wild, R. Ferrini, R. Houdré, M. Mulot, S. Anand, and C. J. M. Smith, Appl. Phys. Lett. 84, 846 (2004).

${ }^{12}$ M. T. Tinker and J. B. Lee, Appl. Phys. Lett. 86, 221111 (2005).

${ }^{13}$ W. Park and J. B. Lee, Appl. Phys. Lett. 85, 4845 (2004).

${ }^{14}$ C. W. Wong, P. T. Rakich, S. G. Johnson, M. Qi, H. I. Smith, E. P. Ippen, L. C. Kimerling, Y. Jeon, G. Barbastathis, and S.-G. Kim, Appl. Phys. Lett. 84, 1242 (2004).

${ }^{15}$ K. Yoshino, Y. Shimoda, Y. Kawagishi, K. Nakayama, and M. Ozaki, Appl. Phys. Lett. 75, 932 (1999).

${ }^{16}$ D. Scrymgeour, N. Malkova, S. Kim, and V. Gopalan, Appl. Phys. Lett. 82, 3176 (2003).

${ }^{17}$ M. Soljacic, S. G. Johnson, S. Fan, M. Ibanescu, E. Ippen, and J. D. Joannopoulos, J. Opt. Soc. Am. B 19, 2052 (2002).

${ }^{18} \mathrm{M}$. Soljacic and J. D. Joannopoulos, Nat. Mater. 3, 211 (2004).

${ }^{19}$ H. Altug and J. Vuckovic, Appl. Phys. Lett. 86, 111102 (2005).

${ }^{20}$ H. Gersen, T. J. Karle, R. J. P. Engelen, W. Bogaerts, J. P. Korterik, N. F. van Hulst, T. F. Krauss, and L. Kuipers, Phys. Rev. Lett. 94, 073903 (2005).

${ }^{21}$ L. Razzari, D. Träger, M. Astic, P. Delaye, R. Frey, G. Roosen, and R. André, Appl. Phys. Lett. 86, 231106 (2005).

${ }^{22}$ F. Lacour, N. Courjal, M.-P. Bernal, A. Sabac, C. Bainier, and M. Spajer, Opt. Mater. (Amsterdam, Neth.) 27, 1421 (2005).

${ }^{23}$ M. Roussey, M.-P. Bernal, N. Courjal, and F. I. Baida, Appl. Phys. Lett. 87, 241101 (2005).

${ }^{24}$ S. Grilli, P. Ferraro, P. De Natale, B. Tiribilli, and M. Vassalli, Appl. Phys. Lett. 87, 233106 (2005).

${ }^{25}$ T. Fujiwara, X. Cao, R. Srivastava, and R. V. Ramaswamy, Appl. Phys. Lett. 61, 743 (1992).

${ }^{26}$ E. L. Wooten, K. M. Kissa, A. Yi-Yan, E. J. Murphy, D. A. Lafaw, P. F. Hallemeier, D. Maack, D. V. Attanasio, D. J. Fritz, G. J. McBrien, and D. E. Bossi, IEEE J. Sel. Top. Quantum Electron. 6, 69 (2000). 